\title{
Identifying Educational Needs of the Multidisciplinary Cancer Team in the Treatment of Metastatic Breast Cancer
}

\author{
Priya Wanchoo, MDª Chris Larrison ${ }^{\mathrm{b}}$; Carol Rosenberg, MD; Naomi Ko, MD, $\mathrm{MPH}^{c}$; \\ Cynthia Cantril, RN, OCN, CBCN, MPH'; Naomi Moellerc; Ruchit Parikh, PharmD; ; and \\ Ana-Marija Djordjevic, PharmD ${ }^{a}$
}

\begin{abstract}
Background: Rapid advancements in the field of metastatic breast cancer $(\mathrm{mBC})$ add to the complexity of managing patients with this disease. An educational needs assessment of multidisciplinary $\mathrm{mBC}$ clinicians was executed to identify practice performance gaps and recommend educational strategies aimed at closing these gaps. Methods: To ensure a collection of reliable data for assessment, a systematic process was used to design, develop, and validate the tools that were used. This grounded theory approach included assessment and confirmation by clinical experts and validation testing within the target audiences. A mixed-methods approach was used to identify practice performance gaps in care, using both qualitative in-depth interviews and quantitative surveying. The quantitative survey assessment consisted of 2 main sections: the Clinician Change Readiness Inventory tool and a Clinical Knowledge and Practice Assessment. Results: The study included 42 clinicians in the interview phase and 186 clinicians in the survey phase from 36 different states. Five key practice performance gaps were identified: (1) selecting optimal treatment, (2) personalizing therapy, (3) monitoring mBC, (4) engaging in effective communication, and (5) balancing patient access and time. Most of the gaps overlap and are related to the integral role communication plays in management decision-making in $\mathrm{mBC}$. Conclusions: Awareness of the key practice performance gaps is critical to inform improvements in quality care.
\end{abstract}

J Natl Compr Canc Netw 2017;15(2):205-212

\section{Background}

Breast cancer $(\mathrm{BC})$ is the most common cancer in women, with an estimated 1.7 million new cases globally each year. ${ }^{1,2}$ Although only $5 \%$ to $10 \%$ of cancers are metastatic at diagnosis, approximately $30 \%$ that are initially diagnosed as early stage will become metastatic, making BC a significant health challenge. ${ }^{1,3}$ When detected early, $\mathrm{BC}$ may be curable; however, metastatic $\mathrm{BC}$ $(\mathrm{mBC})$ is generally considered incurable, with a 5-year survival rate of $25 \%{ }^{3,4}$

A multidisciplinary team (MDT) approach is necessary in treating $\mathrm{mBC}$, in order to provide optimal care

From ${ }^{2}$ Haymarket Medical Education, Paramus, New Jersey; ${ }^{b}$ Healthcare Performance Consulting, Zionsville, Indiana; 'Boston University School of Medicine, Boston, Massachusetts; and 'Sutter Health, Santa Rosa, California. Submitted January 13, 2016; accepted for publication October 17, 2016.

Ms. Cantril has disclosed that she is on the speakers' bureau for Genentech. The remaining authors have disclosed that they have no for the patient. The MDT should consist of a surgical oncologist, radiologist, medical oncologist, pathologist, clinical nurse specialist, and palliative care specialist. ${ }^{5}$ Unfortunately, $59 \%$ of women with BC, particularly $\mathrm{mBC}$, say that most aspects of their lives have changed in a negative way, and that oncologists are not addressing the psychosocial aspects of $\mathrm{BC}$ and are not communicating effectively with patients and caregivers regarding medical treatment, prognosis, healthcare costs, insurance coverage, and quality-of-life and survivorship issues. ${ }^{6}$ According to the National Institutes of Health, a palliative care specialist should be included

financial interests, arrangements, affiliations, or commercial interests with the manufacturers of any products discussed in this article or their competitors.

Correspondence: Priya Wanchoo, MD, Haymarket Medical Education, 140 East Ridgewood Avenue, Suite 370S, Paramus, NJ 07652.

E-mail: priya.wanchoo@haymarketmedical.com 
Wanchoo et al

in the MDT as early as possible to prevent or manage symptoms and side effects of the disease and its treatment, in addition to the related psychological, social, and spiritual problems. ${ }^{7}$ Palliative care at its heart is an MDT approach that focuses on quality of life of patients and their family. Cancer care is undergoing a paradigm shift from disease management to a patient-centered approach that requires patient rights and empowerment. However, palliative care services remain limited. ${ }^{8}$

Primary goals of palliative care include palliation and disease control, with optimal treatment revolving around efficacy and toxicity considerations and patient status and preference. ${ }^{1,9}$ Treatment for $\mathrm{BC}$ is changing, and therapy choices are becoming more individualized for patients. ${ }^{9-13}$ Oncologists feel overwhelmed by the magnitude of and rapidity with which information on molecular testing and new drugs has appeared. Additionally, oncologists are currently unclear about optimizing targeted therapies for patients with early advanced BC. Ongoing research is identifying new treatment paradigms that offer promise to patients. ${ }^{3,14,15}$ Unfortunately, even with these advancements, challenges and performance gaps remain in providing optimal care.

To understand critical knowledge- and competence-based clinical gaps in $\mathrm{mBC}$, we conducted a needs assessment of a cohort of US healthcare professionals (HCPs) who treat patients with $\mathrm{mBC}$. Results provide an analysis of how HCPs are managing $\mathrm{mBC}$ and identify clinical areas that should be addressed in future continuing medical education (CME).

\section{Methods}

A mixed methodology using both qualitative and quantitative research was used for this assessment. To ensure a collection of valid and reliable data for the assessment, a systematic process was used to design, develop, and validate the needs assessment tools. This process included the review of peerreviewed literature, assessment and validation by clinical experts, and validation testing within the target audiences. Beta testing was performed in a 2-phase process. First, the instrument was sent to members of the target audience during interviews to discuss each item, the response chosen by the clinician, and feedback on the question-and-response set. After refinement based on the information learned through the interviews, the assessment was placed online and released to a sample of the target audience to check for adequate item variance, reliability, and convergent/ discriminant validity with respect to other measures.

Repeat sampling was used to ensure a data set representative of the target audiences and results that are generalizable to those audiences. All data collection techniques and processes were adherent to best practices around human research subject protection. Data collected were stored on a locked and password-protected computer, accessible only by authorized study researchers. No private health information was collected. Personal information was collected only for purposes of reimbursing interviewees for their time. This information was separated (de-identified) from the study data. All study data were reported in aggregate fashion only.

The target population was the multidisciplinary clinical team members engaged in the treatment of patients with $\mathrm{mBC}$ at academic medical centers and/or community cancer centers and clinics in the United States. They included oncologists in the academic setting, community oncologists, oncology nurse navigators (ONNs), and oncology nurses.

The needs-assessment methodology included:

- Qualitative interviews: 60-minute telephone interviews were conducted with HCPs with experience managing and treating $\mathrm{mBC}$ in order to identify gaps in knowledge, competence, and performance; key issues and influences on clinical behavior; and decision points in the management of $\mathrm{mBC}$ wherein educational interventions may be helpful. Interview reporting included archetypal responses and key verbatim responses.

- Quantitative clinician survey: this 2-part quantitative assessment was distributed via e-mail invitation. Quantitative assessments underwent statistical analysis, using IBM SPSS Statistics, version 21.0 (IBM Corp, Armonk, NY) to quantify and establish statistical significance of areas such as gaps, attitudes, and barriers. Using estimated response rates based on similar past surveys, partners ensured a minimum of 100 completed surveys for the primary target audience of medical oncologists with repeated sampling. Participants consisted of medical, radiation, and surgical oncologists. The multiplechoice question survey tool took approximately 
Clinical Gaps in Metastatic Breast Cancer

10 minutes to complete and included basic demographic data and 2 main sections:

- Clinician Change Readiness Inventory: designed to assess perceived needs, this tool quantified forces and attitudes around the diagnosis and treatment of $\mathrm{mBC}$ and identified barriers to adherence and clinical best practices. It was organized using clinical competency statements of behaviors essential for best practice related to $\mathrm{mBC}$ care derived from evidence-based clinical practice guidelines with expert input.

- Clinical Knowledge and Practice Assessment: created to assess and quantify actual clinician knowledge as well as practice gaps related to the assessment, diagnosis, and management of $\mathrm{mBC}$, this tool also quantified systems barriers that impede best practices. The multiple-choice questions in the "Practice Assessment" section featured clinical vignettes intended for the primary target medical oncologist audience.

- Expert panel review: on completion of data collection, an advisory panel of clinical experts convened to assist professional educators with the interpretation of these data, suggest further subanalyses, and develop implications for future education.

- Faculty members were selected by Boston University School of Medicine and Haymarket Medical Education based on the faculty's commitment to education and $\mathrm{mBC}$ expertise.

\section{Results}

\section{Sample Demographics}

In-depth interviews were completed with 42 clinicians; 19 from academic medical centers and 23 from community settings. The clinician survey was completed by 186 physicians and 2 nurse practitioners from 36 different states (Table 1); the ONN survey was completed by 13 individuals. Survey participants represented a mix of practice settings (Table 2); $42 \%$ of participants had been in practice $>15$ years.

\begin{tabular}{|c|c|c|c|}
\hline \multicolumn{2}{|c|}{ Interviewees by Profession } & \multicolumn{2}{|c|}{$\begin{array}{l}\text { Survey Participants } \\
\text { by Profession }\end{array}$} \\
\hline Profession & $\begin{array}{l}\text { Number of } \\
\text { Participants }\end{array}$ & Profession & $\begin{array}{l}\text { Number of } \\
\text { Participants }\end{array}$ \\
\hline $\begin{array}{l}\text { Medical } \\
\text { oncologist }\end{array}$ & 17 & $\begin{array}{l}\text { Medical } \\
\text { oncologist }\end{array}$ & 132 \\
\hline $\begin{array}{l}\text { Radiation } \\
\text { oncologist }\end{array}$ & 3 & $\begin{array}{l}\text { Radiation } \\
\text { oncologist }\end{array}$ & 40 \\
\hline $\begin{array}{l}\text { Nurse } \\
\text { practitioner/nurse }\end{array}$ & 14 & $\begin{array}{l}\text { Surgical } \\
\text { oncologist }\end{array}$ & 14 \\
\hline $\begin{array}{l}\text { Oncology nurse } \\
\text { navigator }\end{array}$ & 5 & & \\
\hline Other & 3 & & \\
\hline
\end{tabular}

Practice Performance Gap \#1: Selecting the Optimal Treatment Approach as Patients Progress Throughout the Disease Continuum

An unmet need exists in the ability among medical oncologists to select appropriate therapy options for patients with $\mathrm{mBC}$. Additionally, optimal sequencing of therapies, especially in the metastatic disease setting, remains a challenge. ${ }^{9,10,14}$ The medical oncologists perceived a need in both of those areas, as evidenced by the difference between present and desired ability ratings (Figure 1). These indicate areas in which the physicians desire education in order to change their clinical practice.

The "Practice Assessment" portion of the survey indicates variability in oncologists' abilities to choose appropriate therapy options. Responses to many questions indicated that education is warranted regarding how clinicians think about endocrine therapy and the appropriate patient profiles for this treatment approach. In particular, 19\% of the physicians surveyed indicated that they sometimes try endocrine therapy for estrogen receptor (ER)-negative tumors (Figure 2A), and most of these respondents noted that they would try endocrine therapy before chemotherapy (Figure 2B). This reveals an unmet need in education surround-

\begin{tabular}{|lc|}
\hline Table 2. Survey Participants by Practice Setting \\
\hline Practice Setting & Number of Respondents \\
\hline Academic & 47 \\
\hline Community & 95 \\
\hline Combination academic/community & 31 \\
\hline Free-standing cancer center & 12 \\
\hline Other & 1 \\
\hline
\end{tabular}


Wanchoo et al

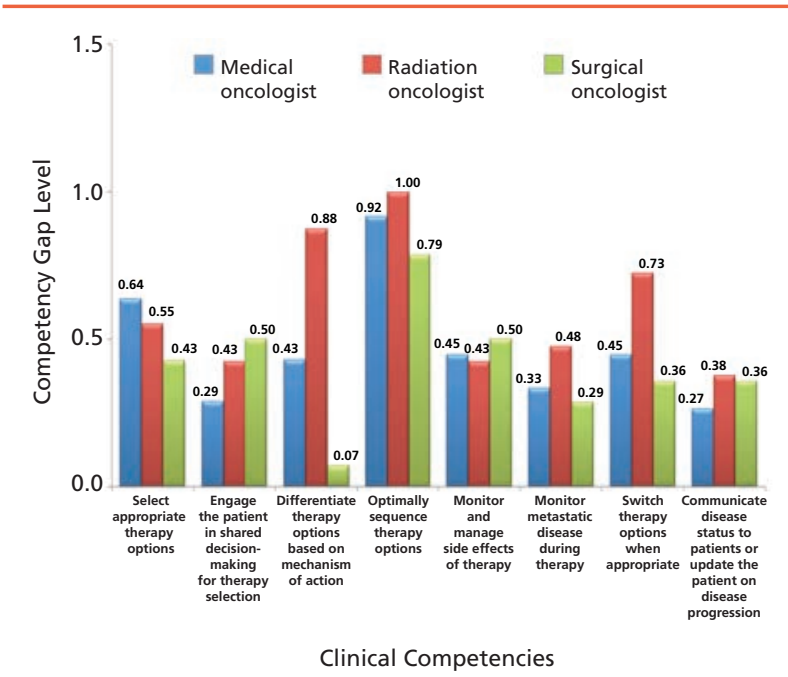

Figure 1. Competency gaps. The average difference, or gap, between the present perceived and desired level of ability rating for each of the competency statements indicates the perceived need of the learners. This gap between the perception of "what is" and "what ought to be" predicts physician motivation to learn and change. When evaluating the medical oncologist group, there are 2 gaps that stand out.

ing how to treat the patient population with ERnegative $\mathrm{mBC}$, specifically in terms of the appropriate patient population to consider for endocrine therapies. Additionally, 19\% of respondents did not select the optimal therapy for a clinical vignette focusing on patients with ER-positive mBC (Figure 3). This clinical vignette requires clinicians to first make a decision between chemotherapy and an antiestrogen therapy. This particular patient should not be prescribed chemotherapy; chemotherapy would be appropriate for a patient with visceral disease. The interviews also revealed a similar deficit in treatment strategies for treating this patient population; ONNs indicated that some clinicians are quick to treat with chemotherapy when managing this patient population. Educating clinicians on the rationale behind the main tenet of treatment for patients with ER-positive $\mathrm{mBC}$, which is to start with an antiestrogen first, is critical.

Additional information regarding education on therapies was gleaned from the needs assessment. When asked to list the most important topics to cover in $\mathrm{mBC}$ education, $>75 \%$ of the medical oncologists indicated therapy-related topics. The NCCN Clinical Practice Guidelines in Oncology for Breast Cancer were reported to be the most trusted source for treatment recommendations. Although $61 \%$ of respondents said that the NCCN Guidelines provide just the right amount of information, $34 \%$ said that they would like more guidance, which further supports the desire for more education.

\section{Practice Performance Gap \#2: Personalizing Treatment Strategies Based on Individual Patient Characteristics}

Medical oncologists exhibit educational need when making treatment decisions for patient subpopulations, including premenopausal and postmenopausal women, patients with comorbidities, and other patients who may present unique $\mathrm{mBC}$ challenges. Results of the clinical vignette challenges are suggestive of a larger issue focused on a potential deficit in clinicians' abilities to take into consideration individual patient characteristics when making treatment decisions. This Practice Performance Gap shares similarities with Practice Performance Gap \#1.

Medical oncologists may not consider the entire patient's clinical condition, including comorbidities, when evaluating possible treatment strategies. As will be described in Practice Performance Gap \#3, side effects are a focus of interest for patients with $\mathrm{mBC}$. However, when asked for recommended treatments for an already sick patient experiencing pulmonary complications, $18 \%$ of medical oncologists chose a

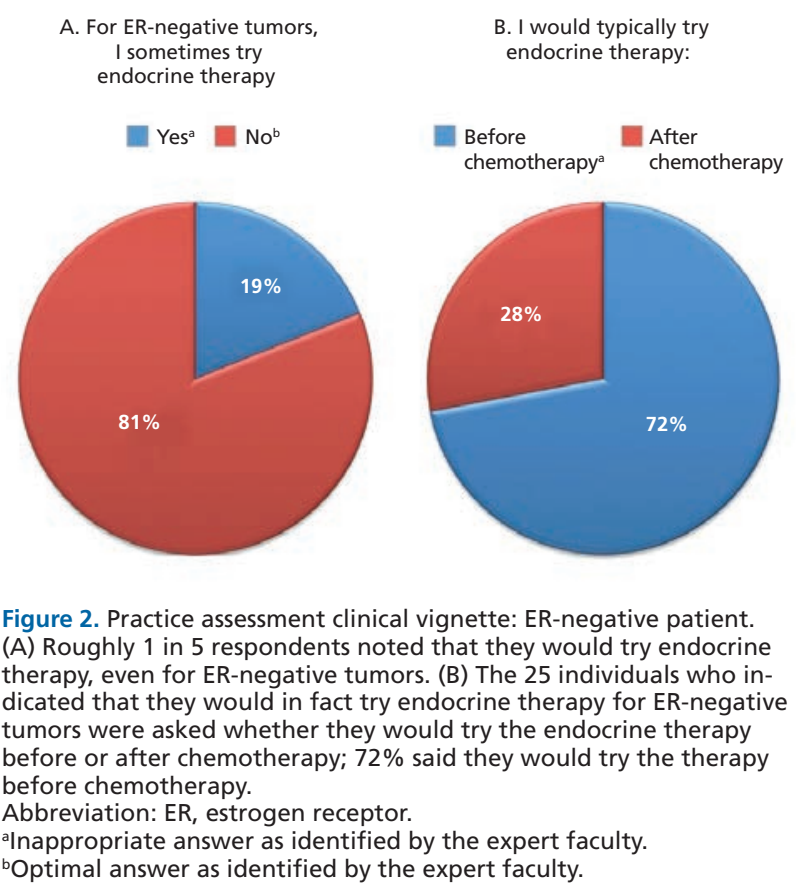


treatment associated with pulmonary complications (Figure 4). Thus, there is an opportunity for education concerning the impact of treatment-related side effects as they relate to individual patients, such as regarding quality of life; results from this clinical vignette are not reflective of a prioritization of side effect implications. Additionally, an opportunity was identified for education focusing on an individualized approach to patient care, balancing toxicities with quality of life, and other options. When asked which treatment they would recommend for a very fragile person with indolent disease and toxicities from her previous initial therapy, 1 in 4 clinicians chose an aggressive approach (Figure 5). Despite multiple options to choose from and no urgencies due to her disease status, $23 \%$ of medical oncologists chose a treatment strategy that seemingly did not balance the patient's quality of life with toxicity considerations.

\section{Practice Performance Gap \#3: Appropriately and Effectively Monitoring Metastatic Disease, Including Disease Progression and Side Effects} Although results from the "Competency Gaps" analysis suggest that there is not a perceived need in monitoring and managing the side effects of therapy or in monitoring metastatic disease during therapy (Figure 1), clinical evaluation by the expert panel suggested otherwise. For instance, 55\% of medical oncologists indicated that they would obtain a PET/CT scan when there was in fact no role for a PET/CT in an asymptomatic individual. Further expert discussion indicated that information on monitoring disease, including evidence-based radiologic studies and $\mathrm{BC}$ disease surveillance, presents an area of educational need.

A critical aspect of patient-physician communication is the discussion of side effects. Although respondents in this study averaged low agreement with the statement "My patients do not always report the side effects they experience," critical expert analysis supports the need for enhanced effective communication between physicians and patients when it comes to side effects. The interview portion of the study also revealed that patient reporting of side effects was a concern; this was reported more by ONNs than physicians. There are indications that patients may not be communicating side effects to their physicians for fear that their oncologist may stop their medication. ${ }^{1,16,17}$ Thus, physician aware-
A 64-year-old obese but otherwise well female (PS, 0) presents with newly diagnosed left breast cancer that is ER-positive, PR-positive, and HER2-negative. Staging is remarkable for CEA 15 and CT chest/abd/pelvis and bone scan is negative except 3 subtle bone lesions in the femur. The biopsy is positive for metastasis and she receives a diagnosis of stage IV disease [T2N2M1]. What treatment would you mostoften recommend along with biphosphonates?

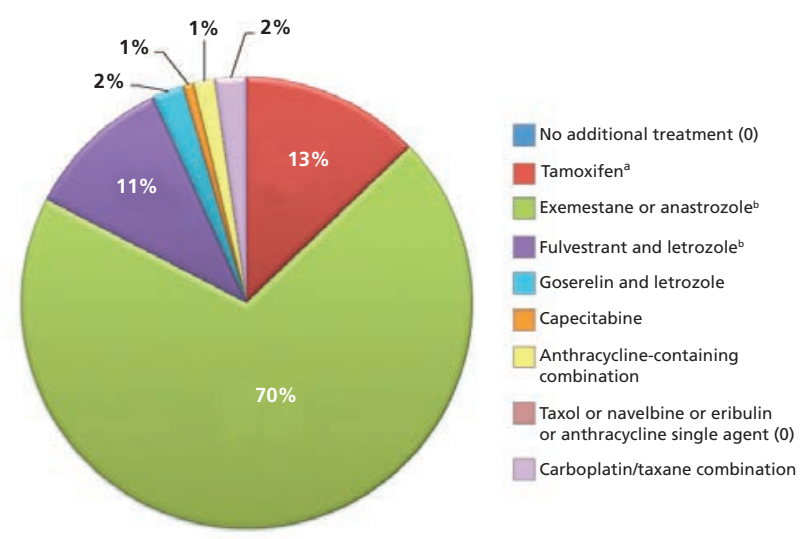

Figure 3. Practice assessment clinical vignette: ER-positive patient. Although $94 \%$ of respondents appropriately chose an endocrine therapy, nearly 1 in 5 did not select the optimal therapy as determined by the expert panel (exemestane, anastrozole, or fulvestrant + letrozole). Abbreviations: abd, abdomen; CEA, carcinoembryonic antigen; ER, estrogen receptor; PR, progesterone receptor; PS, performance status. ${ }^{a}$ Acceptable answer as identified by the expert faculty.

${ }^{b}$ Optimal answer as identified by the expert faculty.

ness surrounding the deficit in patient reporting of side effects may be lacking and must be emphasized with practicing oncologists.

\section{Practice Performance Gap \#4: Engaging in Effective Patient-Physician Communication, Especially in the Context of Difficult Discussions}

Effective physician-patient communication ensures realistic expectations about interventions, prognosis, and outcomes. The Clinical Competencies exercise measured present and desired abilities of clinicians in order to identify perceived competency gaps (Figure 1). Interestingly, the smallest perceived gaps in ability were related to engaging the patient in shared decision-making for therapy selection and communicating disease status or updating patients on disease progression, which were the only 2 competencies related to patient-physician communication. Furthermore, respondents strongly agreed that their patients were involved in therapy decisions. Although there is low perceived need, analysis by the expert clinical panel and evaluation of the current literature revealed an educational need for improvement surround- 
Wanchoo et al

A 63-year-old with ER-and PR-positive [low] breast cancer affecting the liver and mediastinum is now progessing after letrozole and tamoxifen, with new pulmonary metastases causing cough and shortness of breath and rising liver function tests. What treatment would you recommend?
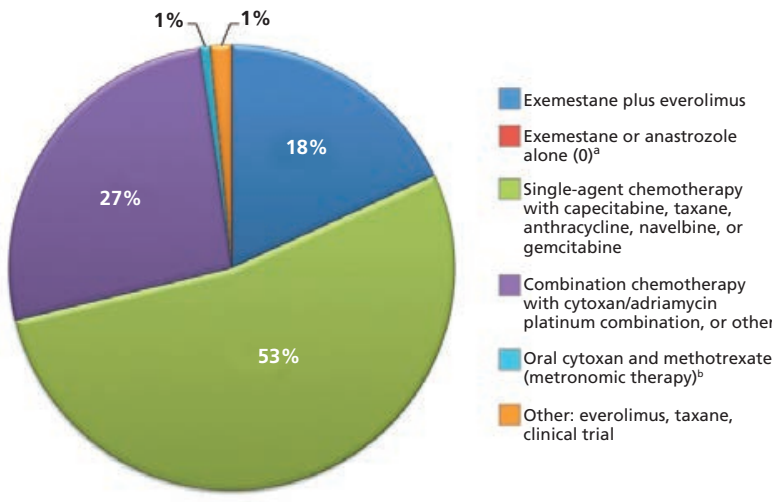

Figure 4. Practice assessment clinical vignette: patient with pulmonary complications. Collectively, $80 \%$ of respondents indicated they would treat with either single-agent or combination chemotherapy; $18 \%$ said they would treat with exemestane plus everolimus.

Abbreviations: ER, estrogen receptor; PR, progesterone receptor.

annappropriate answer as identified by the expert faculty.

'Optimal answer as identified by the expert faculty.

ing patient-physician communication and the model of shared decision-making. ${ }^{1,17-19}$ Education on successful communication approaches, such as in prognostic discussions, and how to include accompanying empathic strategies is key. ${ }^{16}$

Additionally, the lowest barrier to clinical practice as determined by this needs assessment survey was "Lack of patient education materials"; yet further clinical expert analysis concluded that there is an unmet need for more and improved patient resources, as supported by data from recent patient surveys. ${ }^{1,20-23}$

\section{Practice Performance Gap \#5: Managing Obstacles Such as Patient Access and Inadequate Time as a Resource}

Patient access and a lack of time as a resource were identified as barriers to adopting new therapies in clinical practice. The time, attention, and energy needed to manage patients with $\mathrm{mBC}$ can be overwhelming for a medical oncologist. Other members on the MDT, such as the palliative care specialist, would better serve in guiding improvement in quality of life for patients. Issues in this area include pain and psychological, psychosocial, and often comorbid conditions.

Medical oncologists rated high cost to patients, patients' lack of insurance coverage, and lack of time to learn about new therapies as their top 3 barriers to adopting new therapies, suggesting that patientaccess issues are a significant concern in $\mathrm{mBC}$ care. Patient access is also important to patients. ${ }^{20,23}$ The busy schedules of medical oncologists is revealed by the barrier ranked third, which indicates that time is of the essence and that clinicians often feel they do not have enough time to stay abreast of the rapidly changing environment.

\section{Discussion}

This needs assessment illuminates both the real and perceived needs of clinicians treating and managing patients with $\mathrm{mBC}$. Physicians indicated a strong desire for competence in managing these patients, and will be motivated to learn and change if shown evidence of real needs within the content of an educational program. Although the medical oncologists are, in general, very confident and have few perceived needs, this study demonstrates that rapid advancements in the field of $\mathrm{mBC}$ and the oncology field as a whole continually present an educational need and stimulate interest in learning.

Physicians are encouraged to change clinical behavior based on the presence or absence of various forces in their environment. ${ }^{20}$ The Forces for Change analysis underscores the dedication of the clinician population toward learning and education in the topics described. The highest forces for change were the professional forces: "New information in the literature has increased my interest in adopting new practices in this area," and "I wish I were more knowledgeable in this area." There is always new information in the area of $\mathrm{mBC}$; thus, education with this focus is supported and desired by HCPs in this field of medicine. When asked how confident the oncologists were in their ability to remain on the cutting edge with regard to $\mathrm{mBC}$ treatment, interestingly, confidence showed a negative correlation with knowledge: those who answered fewer of the treatment-decision questions correctly had a higher level of confidence in their treatment decisions. Thus, confidence is not a good predictor of knowledge in this area. When considering continuing education, outcomes metrics should be tied to actual knowledge or performance measures and not to confidence.

Improving patient-physician communication skills is a common theme that has emerged as the 
A 78-year-old with COPD oxygen dependent/DM/HTN/CAD/CKD/arthritis/GERD, had ER-positive breaast cancer treated in adjuvant setting with surgery, radiation, and tamoxifen. Fifteen years later, rib films that were done due to a mechanical fall revealed multiple pulmonary nodules. Results from a fine needle aspiration are consistent with metastatic cancer that is grade 2 and ER-positive. She receives anastrozole. After 6 months her arthralgias are noticeably worse and her nodules are stable. What treatment would you recommend?

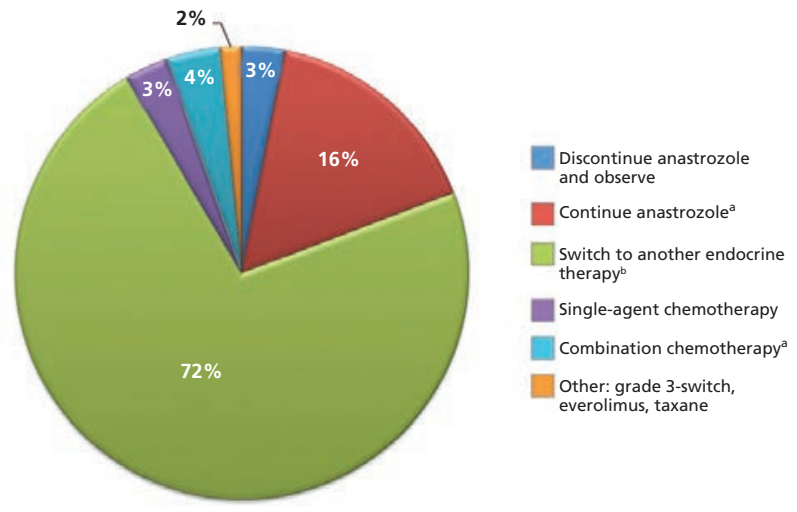

Figure 5. Practice assessment clinical vignette: patient with comorbidities. Although $72 \%$ of respondents noted that they would switch this patient to another endocrine therapy, $16 \%$ would continue anastrozole and $7 \%$ would administer either single-agent or combination chemotherapy. Thus, 1 in 4 individuals would not make the optimal choice.

Abbreviations: CAD, coronary artery disease; CKD, chronic kidney disease; COPD, chronic obstructive pulmonary disease; DM, diabetes mellitus; ER, estrogen receptor; GERD, gastroesophageal reflux disease; HTN, hypertension.

anappropriate answer as identified by the expert faculty.

boptimal answer as identified by the expert faculty.

foundation of the educational strategy recommended for future CME interventions. Embedding the topics of communication and patient engagement into patient case presentations is recommended to emphasize the importance of taking a holistic approach to patient care. In this instance, integration of the patient voice may be most successful in communicating this concept. Suggested approaches to education include integration of patient perspectives into clinical cases and emphasis on an MDT approach to disease management, including the incorporation of nurse navigators. Creating patient engagement tools that aid in decision-making can facilitate more productive conversations. Benefits of focusing on the concept of patient education include the potential to alleviate the burdens on the physician, minimize patient misinformation, and improve patient satisfaction and outcomes.
Future CME should include a modeling forum or clinical vignettes. Patient cases are complex-there is an ongoing need for physicians to be exposed to multifaceted clinical challenges. This approach may be executed through a small-group educational format using teaching cases, similar to tumor boards; however, patient case scenarios also lend themselves well to symposia and text-based cases featuring video vignettes. When educating clinicians about therapies, content should cover efficacy, side effects, placement in a sequencing regimen, and mechanisms of action. Where appropriate, financial implications of therapies should also be covered to provide clinicians an idea of potential patient-access challenges. For all educational initiatives, outcomes metrics should be tied to actual knowledge or performance measures and not to confidence, because this needs assessment demonstrated that confidence is not a good predictor of knowledge in $\mathrm{mBC}$.

Integration of individuals such as ONNs into an MDT approach may help alleviate some of the communication burdens on practicing oncologists. Some ONNs also advocate for the integration of peer-topeer patient programs in an $\mathrm{mBC}$ setting, which may even further alleviate these challenges. ${ }^{1,2,10}$ However, integration of ONNs and/or patient peers is not meant to replace the role of the practicing oncologist, but rather to augment the physician and, ultimately, offer a fully multidisciplinary approach to care.

\section{Conclusions}

Five key practice performance gaps and associated needs in the $\mathrm{mBC}$ academic- and community-based settings have been identified from this research. These gaps describe issues that can impact clinical efficiencies and, ultimately, the quality patient care and outcomes. Each of the key practice performance gaps is addressable from an educational perspective. HCPs treating $\mathrm{mBC}$ exhibit a desire to learn and significant readiness to change if shown evidence of benefits. Overall, pertinent, high-quality, evidencebased education that meets identified needs will ensure that HCPs continually improve their practice, ultimately resulting in better care and optimal outcomes in patients with $\mathrm{mBC}$. 
Wanchoo et al

\section{References}

1. Cardoso F, Costa A, Norton L, et al. ESO-ESMO 2nd international consensus guidelines for advanced breast cancer (ABC2). Ann Oncol 2014;25:1871-1888.

2. Latest world cancer statistics. Global cancer burden rises to 14.1 million new cases in 2012: marked increase in breast cancers must be addressed [press release]. Lyon/Geneva: International Agency for Research on Cancer; December 12, 2013. Available at: https://www. iarc.fr/en/media-centre/pr/2013/pdfs/pr223_E.pdf. Accessed December 29, 2015.

3. Gucalp A, Gupta GP, Pilewskie ML, et al. Advances in managing breast cancer: a clinical update. F1000Prime Rep 2014;6:66.

4. American Cancer Society. Cancer Facts \& Figures 2015. Available at: http://www.cancer.org/acs/groups/content/@editorial/documents/ document/acspc-044552.pdf. Accessed December 29, 2015

5. Abdulrahman GO Jr. The effect of multidisciplinary team care on cancer management. Pan Afr Med J 2011;9:20.

6. Mayer M, Huñis A, Oratz R, et al. Living with metastatic breast cancer: a global patient survey. Commun Oncol 2010;7:406-412.

7. Palliative Care in Cancer. National Cancer Institute Web site. Available at: http://www.cancer.gov/about-cancer/advanced-cancer/ care-choices/palliative-care-fact-sheet. Published March 16, 2010. Accessed September 9, 2016.

8. Davis MP, Strasser F, Cherny N. How well is palliative care integrated into cancer care? A MASCC, ESMO, and EAPC Project. Support Care Cancer 2015;23:2677-2685

9. Partridge $\mathrm{AH}$, Rumble RB, Carey LA, et al. Chemotherapy and targeted therapy for women with human epidermal growth factor receptor 2-negative (or unknown) advanced breast cancer: American Society of Clinical Oncology clinical practice guideline. J Clin Oncol 2014;32:3307-3329.

10. Van Poznak C, Somerfield MR, Bast RC, et al. Use of biomarkers to guide decisions on systemic therapy for women with metastatic breast cancer: American Society of Clinical Oncology clinical practice guideline. J Clin Oncol 2015;33:2695-2704.

11. Burstein HJ, Temin S, Anderson H, et al. Adjuvant endocrine therapy for women with hormone receptor-positive breast cancer: American Society of Clinical Oncology clinical practice guideline focused update. J Clin Oncol 2014;32:2255-2269.

12. Giordano SH, Temin S, Kirshner JJ, et al. Systemic therapy for patients with advanced human epidermal growth factor receptor 2 positive breast cancer: American Society of Clinical Oncology clinical practice guideline. J Clin Oncol 2014;32:2708-2099.
13. Ramakrishna N, Temin S, Chandarlapaty S, et al. Recommendations on disease management for patients with advanced human epidermal growth factor receptor 2-positive breast cancer and brain metastases: American Society of Clinical Oncology clinical practice guideline. J Clin Oncol 2014;32:2100-2108.

14. Kaufman B, Shapira-Frommer R, Schmutzler RK, et al. Olaparib monotherapy in patients with advanced cancer and a germline BRCA1/2 mutation. J Clin Oncol 2015;20:244-250.

15. Martin M, Beslija S, Carrasco E, et al. Phase III study of palbociclib in combination with exemestane vs. capecitabine, in hormonal receptor (HR) positive/HER2 negative metastatic breast cancer (MBC) patients with resistance to non-steroidal aromatase inhibitors (NSAI): PEARL study (GEICAM/2013-02_CECOG/BC.1.3.006) [abstract]. Ann Oncol 2014;25(Suppl 4):iv134-135. Abstract 409TiP.

16. The Psychological Impact of Advanced Breast Cancer. Available at: https://www.novartisoncology.com/sites/www.novartisoncology.com/files/ MYDC_2014SurveyResults_Infographic_w01_0.pdf. Accessed January 3, 2017.

17. Stein J. Better communication needed between breast cancer patients and professionals. Available at: https://www.novartisoncology.com/sites/ www.novartisoncology.com/files/MYDC_2014SurveyResults_Infographic w01_0.pdf. Accessed January 3, 2017.

18. Pfizer and breast cancer leaders join together to increase understanding and dispel myths about metastatic breast cancer [press release]. New York, NY: Pfizer; October 8, 2014. Available at: http://www.pfizer.com/ news/press-release/press-release-detail/pfizer_and_breast_cancer_leaders_ join_together_to_increase_understanding_and_dispel_myths_about_ metastatic_breast_cancer. Accessed February 10, 2015.

19. In-Office Ethnographic Study, sponsored by Pfizer Oncology. April 2014.

20. Mayer M. Lessons learned from the metastatic breast cancer community. Semin Oncol Nurs 2010;26:195-202.

21. Stenger M. Most patients do not report that cure is highly unlikely with chemotherapy for advanced cancer. Available at: http://www.ascopost. $\mathrm{com} /$ issues/february-1,-2013/most-patients-do-not-report-that-cure-ishighly-unlikely-with-chemotherapy-for-advanced-cancer.aspx. February 27, 2015.

22. Weeks JC, Catalano PJ, Cronin A, et al. Patients' expectations about effects of chemotherapy for advanced cancer. N Engl J Med 2012;367:1616-1625.

23. Mayer M, Hunis A, Oratz R, et al. Living with metastatic breast cancer: a global patient survey. Community Oncol 2010;7:406-412. 